\title{
Burden and Disease Characteristics of Patients with Psoriatic Arthritis: A Population-based Cross-sectional Study
}

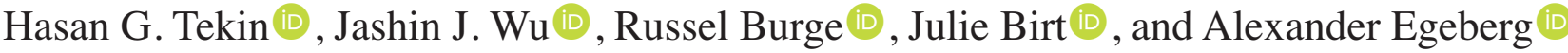

\begin{abstract}
Objective. To describe the prevalence and treatment regimes, disease characteristics, and comorbid diseases among patients with psoriatic arthritis (PsA) in Denmark.

Methods. All Danish individuals aged $\geq 18$ years with rheumatologist-diagnosed PsA were linked in nationwide administrative registers.

Results. Among 4.7 million individuals in Denmark, 10,577 patients with PsA had been diagnosed by a rheumatologist. A female predominance (54.5-59.8\%) was seen among patients with PsA, and about half of the patients $(53.0 \%)$ had received no treatment or treatment only with nonsteroidal antiinflammatory drugs/systemic corticosteroids, while $32.9 \%$ had received nonbiological disease-modifying antirheumatic drugs (DMARD) and $14.1 \%$ had been treated with biologicals. Cutaneous psoriasis was recorded in $66.2-72.3 \%$ of patients with PsA, and patients with severe PsA had the highest prevalences of distal interphalangeal arthropathy, spondylitis, and arthritis mutilans. Smoking and comorbid diseases such as hypertension, diabetes, depression, and anxiety were seen frequently in patients with PsA, but did not significantly differ across severities of PsA.

Conclusion. Disease burden appeared to be significant in patients with PsA across all severities. A considerable proportion of patients with PsA did not receive active antipsoriatic treatment, and about 1 out of 3 patients was not diagnosed with psoriasis. Cutaneous symptoms of psoriasis in patients with PsA might be either underreported or undertreated. (First Release March 1 2019; J Rheumatol 2019;46:716-20; doi:10.3899/jrheum.180670)
\end{abstract}

Key Indexing Terms:

PSORIATIC ARTHRITIS

PSORIASIS

DISEASE BURDEN

EPIDEMIOLOGY

Psoriasis is a chronic T cell-mediated inflammatory disease seen in $2-3 \%$ of the population worldwide ${ }^{1}$.

Psoriatic arthritis (PsA) is an inflammatory spondyloar-

From the Department of Dermatology and Allergy, Herlev and Gentofte Hospital, University of Copenhagen, Hellerup, Denmark; Kaiser Permanente Los Angeles Medical Center, Department of Dermatology, Los Angeles, California; Eli Lilly and Co., Indianapolis, Indiana; Division of Pharmaceutical Sciences, University of Cincinnati, Cincinnati, Ohio, USA.

This study was funded by Eli Lilly and Co. The funding source participated in interpretation of the final analyzed study results, but had no access to the raw data, and did not participate in data collection, management, or analysis. Dr. Wu is an investigator for AbbVie, Amgen, Eli Lilly, Janssen, Novartis, and Regeneron. Dr. Burge and J. Birt are currently employed by Eli Lilly and Co. Dr. Egeberg has received research funding from Pfizer, Eli Lilly, the Danish National Psoriasis Foundation, and the Kgl Hofbundtmager Aage Bang Foundation, and honoraria as a consultant and/or speaker from Leo Pharma, Samsung Bioepis Co. Ltd., Pfizer, Eli Lilly, Novartis, Galderma, and Janssen Pharmaceuticals.

H.G. Tekin, MD, Department of Dermatology and Allergy, Herlev and Gentofte Hospital, University of Copenhagen; J.J. Wu, MD, Kaiser Permanente Los Angeles Medical Center, Department of Dermatology; R. Burge, PhD, Eli Lilly and Co., and Division of Pharmaceutical Sciences, University of Cincinnati; J. Birt, PharmD, Eli Lilly and Co.; A. Egeberg, MD, PhD, Department of Dermatology and Allergy, Herlev and Gentofte Hospital, University of Copenhagen.

Address correspondence to Dr. A. Egeberg, Department of Dermatology and Allergy, Herlev and Gentofte Hospital, Kildegårdsvej 28, 2900

Hellerup, Denmark.E-mail: alexander.egeberg@gmail.com

Accepted for publication October 23, 2018. thropathy ${ }^{2}$ seen in 30\% of patients with psoriasis ${ }^{3}$. In 1973 PsA was divided by Moll and Wright into 5 subtypes: an oligoarticular disease with $\leq 5$ affected joints, a polyarticular disease with involvement of $>5$ joints, a form that only affects distal interphalangeal (DIP) joints, an axial subtype with spondylitis or sacroiliitis, and a severe erosive form called arthritis mutilans ${ }^{4}$. In recent years, the consensus has been that any of these subtypes can be present in different combinations and that the pattern of the disease can change and progress over time. The heterogeneity of PsA and lack of good diagnostic tests has made it challenging to study and to reproduce epidemiological data, although recent information suggests an increasing incidence of PsA diagnosed in the general population ${ }^{5}$. Most prior studies investigating disease characteristics of PsA are based on smaller observational studies, and in more recent years only a few larger population-based studies have investigated the prevalence of disease characteristics and comorbidities in the general population $6,7,8$. The prevalence of comorbidities in PsA across the severity of the disease is not well described. The aim of our present study was to describe the demographics, comorbidities, and clinical characteristics of patients with PsA in the general population of Denmark across disease severities. 


\section{MATERIALS AND METHODS}

Danish law does not require ethics approval for use of administrative data. Data sources. All citizens in Denmark have a personal identification number, which is assigned at birth or at migration into the country. The personal identification number makes it possible to link citizens in different nationwide registers using the Civil Registration System, which contains data on sex, birth, death, and migration ${ }^{9}$. The Danish National Patient Register contains data on diagnosis and procedures (e.g., biologic therapy given at outpatient clinics) from hospital admissions, ambulatory consultations, and from a number of private clinics. The diagnosis of PsA in The Danish National Patient Register was recorded according to the Danish adaptations of the International Classification of Diseases, 8th revision (ICD-8) until 1994 and afterward to ICD- $10^{10}$. According to the Danish version of the ICD system, specific information on certain subtypes of PsA are recorded in the registers including DIP joint involvement, spondylitis, and arthritis mutilans, although specific data on other subtypes such oligoand polyarthritis are not registered. Such patients are thus simply classified as having PsA without subtype specification.

Data on pharmacy-dispensed medication, including quantity and formulation, are registered in the Danish Registry of Medicinal Products Statistics according to the Anatomical Therapeutic Chemical classification ${ }^{11}$. Tax-reported earnings from all Danish residents (herein used for calculation of socioeconomic status) are registered in the Income Statistics Register ${ }^{12}$. Consultations in private and public clinics, including with physiotherapists, chiropractors, and psychologists, and covered by private and public health insurance, are registered by Statistics Denmark in the Health Care Statistics Register ${ }^{13}$.

Study population. We performed a nationwide cross-sectional study of all individuals aged 18 years or older who were alive and were residents of Denmark on December 31, 2015. Unless otherwise specified, data were collected from database inception until that date. The prevalence of PsA was estimated on diagnosis regardless of the physicians' specialty; however, all other analyses on PsA and its characteristics were made on rheumatologist-diagnosed PsA, to ensure accuracy of the diagnosis. Using a validated algorithm, patients were divided into subgroups of mild, moderate, and severe disease, regarding the type of treatment they had received ${ }^{14}$. Consequently, patients receiving nonsteroidal antiinflammatory drugs (NSAID), systemic corticosteroids, or no treatment at all were classified as having mild PsA. Patients were considered to have moderate PsA if they received treatment for PsA with disease-modifying antirheumatic drugs [DMARD; sulfasalazine, leflunomide, cyclosporine (CSA), or methotrexate (MTX)], and severe PsA if they received biologics as treatment for their PsA [adalimumab (ADA), certolizumab, etanercept (ETN), golimumab, infliximab (IFX), and ustekinumab] or the phosphodiesterase-4 inhibitor apremilast (for simplicity these are henceforth referred to as "biologics"). Similarly, cutaneous psoriasis was classified as mild if patients received no treatment or only topical therapy. Patients receiving systemic nonbiologic therapy for psoriasis (i.e., MTX, CSA, retinoids, and phototherapy) were classified as having moderate disease, and patients were considered to have severe disease if they had been treated for psoriasis with biologics (ETN, IFX, ADA, ustekinumab, or apremilast).

Information on smoking was performed using a data-retrieval algorithm as previously described ${ }^{15}$. To include patients in the PsA population with cutaneous psoriasis that may not have been formally diagnosed, the "any psoriasis" assessment was made by either a diagnosis of psoriasis or use of topical vitamin D analogs, the preferred first-line therapy for psoriasis in Denmark and used exclusively for this condition ${ }^{16}$. For all other analyses, psoriasis was limited to diagnostic (ICD) codes.

Statistical analysis. Summary statistics were generated and expressed as means with SD for normally distributed variables, median and interquartile ranges for non-normally distributed continuous variables, and frequencies for categorical variables. Results were described for PsA, psoriasis, and subgroups based on disease severity, and compared between groups, with Pearson chi-square test for dichotomous variable comparisons. ANOVA was performed where applicable. $\mathrm{P}$ values $<0.05$ were considered statistically significant. All analyses were performed using SAS statistical software version 9.4 (SAS Institute Inc.) and STATA software version 13.0 (StataCorp).

\section{RESULTS}

The Danish population comprised 4,689,946 adults on December 31, 2015, including 14,857 patients with PsA, yielding an overall prevalence of $0.32 \%$. Among these patients, 71.2\% $(\mathrm{n}=10,577)$ had been diagnosed by a rheumatologist, yielding a rheumatologist-verified PsA prevalence of $0.22 \%$. Among patients with rheumatologist-verified PsA, 53.0\% $(\mathrm{n}=5611)$ had mild PsA, 32.9\% $(\mathrm{n}=3479)$ had moderate PsA, and $14.1 \%(\mathrm{n}=1487)$ had severe PsA. Overall, the PsA population had a female predominance ranging between $54.5 \%$ and $59.8 \%$ across severities. The mean age was between 53.7 and 57.9 years, with a mean disease duration of 8.9-10.5 years (Table 1).

Based on available data, DIP joint involvement was the most frequent subtype and was seen in $8.3-16.8 \%$ of patients, followed by arthritis mutilans (seen in $2.8-9.9 \%$ of patients) and psoriatic spondylitis (seen in $2.2-7.3 \%$ of patients). Importantly however, data on other subtypes were not available for our study. Within the past 12 months, 19.1-29.7\% of patients with PsA had been treated by a physiotherapist, whereas 5.3-7.9\% had received chiropractic treatments, and $0.9-3.6 \%$ had been treated by a private-practicing rheumatologist. Use of acupunctural treatments was very infrequent and did not significantly differ across severities.

Psoriasis, regardless of diagnosing physician, was recorded in $66.2-72.3 \%$ of patients with PsA, whereas dermatologist-diagnosed psoriasis was present in $19.8-35.7 \%$ of patients (Table 1). Overall, patients with DIP joint involvement, arthritis mutilans, and psoriatic spondylitis were more frequently classified with severe PsA and treated with systemic or biologic therapy. Patients with severe PsA had the highest recorded psoriasis prevalence (35.7\%), whereas $19.8 \%$ and $27.7 \%$ of patients with moderate and mild PsA, respectively, also were diagnosed with psoriasis. Conversely, PsA was seen in 16.3-47.9\% of patients with psoriasis, and rheumatologist-verified PsA was seen in $11.4-36.9 \%$ of patients with psoriasis (Table 2).

There was a considerable psychological burden in patients with PsA, and use of antidepressants occurred in 12.6-13.5\% of patients, although use of anxiolytics and antipsychotics was less frequent (Table 1). Potent antidepressants such as tricyclic antidepressants were used by $4.0-5.1 \%$ of patients with PsA, and $1.5-3.2 \%$ and $1.3-1.8 \%$ of patients had consulted a psychologist and psychiatrist, respectively, within the past year.

Interestingly, diabetes and hypertension (HTN) were significantly more frequent among psoriasis patients with concurrent PsA.

Personal non-commercial use only. The Journal of Rheumatology Copyright @ 2019 . All rights reserved. 
Table 1. Demographic characteristics and comorbidities of PsA by severity.

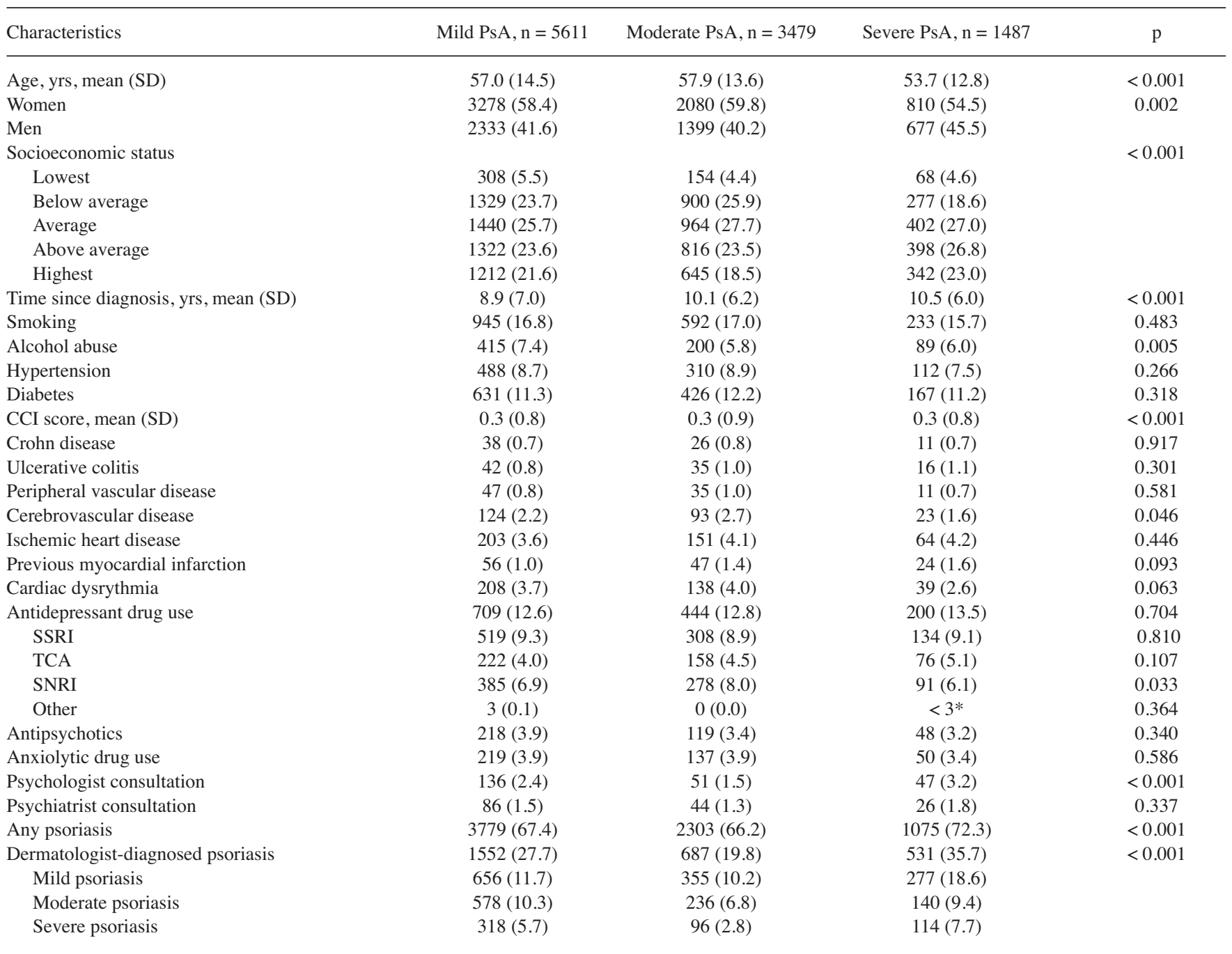

Data are n (\%) unless otherwise specified. * Data shown as < 3 because of data security requirements. PsA: psoriatic arthritis; CCI: Charlson Comorbidity Index; SSRI: selective serotonin reuptake inhibitors; TCA: tricyclic antidepressants; SNRI: serotonin norepinephrine reuptake inhibitor.

Table 2. Prevalence of PsA among patients with psoriasis.

\begin{tabular}{lcccc}
\hline Variables & \multicolumn{3}{c}{ Psoriasis } \\
& Mild, $\mathrm{n}=11,341$ & Moderate, $\mathrm{n}=6690$ & Severe, $\mathrm{n}=1431$ & $\mathrm{p}$ \\
\hline Any diagnosis of PsA & $1845(16.3)$ & $1359(20.3)$ & $685(47.9)$ & $<0.001$ \\
Rheumatologist-diagnosed PsA & $1288(11.4)$ & $954(14.3)$ & $528(36.9)$ & $<0.001$ \\
Mild PsA & $656(5.8)$ & $578(8.6)$ & $318(22.2)$ & \\
Moderate PsA & $355(3.1)$ & $236(3.5)$ & $96(6.7)$ & \\
Severe PsA & $277(2.4)$ & $140(2.1)$ & $114(8.0)$ & \\
\hline
\end{tabular}

Data are $\mathrm{n}(\%)$. PsA: psoriatic arthritis.

\section{DISCUSSION}

In this nationwide population-based cross-sectional study, the overall prevalence of PsA was $0.32 \%$ in the general population, regardless of the diagnosing physician's specialty. In analyses restricted to patients with rheumatologist-verified PsA, about half of these received no treatment or treatment with only NSAID/systemic corticosteroids, while the other half received mainly DMARD with or without biologicals. Psoriasis was diagnosed in about 2 out of 3 patients with PsA, and severe PsA was more associated with severe psoriasis, and vice versa. 
The prevalence of PsA in Denmark has been described previously ${ }^{5}$, and there seems to be an increasing incidence in recent years. As in previous studies ${ }^{2,6}$, we found a female predominance that was present across disease severities. In accordance with previous studies ${ }^{6}$, patients with PsA were in their sixth decade of life, with a disease duration of 8-11 years, meaning that patients on average were diagnosed in their fifth decade. Around half of patients did not receive any treatment or were treated only with NSAID/systemic corticosteroids. Similar to this, Ogdie and colleagues ${ }^{6}$ reported data from a large population-based study in the United Kingdom, in which about half of the patients received DMARD while the other half were mainly untreated. However, in that study, data were limited to prescriptions made by general practitioners, thus excluding treatment given in hospital clinics or from private rheumatologists and dermatologists, whereas such data were included in our study, yielding treatment data that were more complete. Nonetheless, these findings suggest a possible undertreatment of psoriasis and PsA symptoms in a considerable proportion of patients with these diseases. Patients with severe PsA had a higher prevalence of all PsA subtypes (i.e., DIP joint affection, spondylitis, and arthritis mutilans), and a higher proportion of patients were also treated by chiropractors and physiotherapists compared with patients having mild or moderate PsA. To our knowledge, such data have not previously been described. The overall prevalence of the 3 reported PsA subtypes in our study appear to be in agreement with previously reported numbers, although historical estimates vary widely ${ }^{17}$.

The burden of disease appears to be significant in patients with PsA, and $42 \%$ are reported to have 3 or more comorbid conditions ${ }^{18}$. We found that smoking was strongly associated with PsA and was identified as a risk factor by some studies, while other studies have suggested an inverse association ${ }^{19}$. Along these lines, we found a higher prevalence of diabetes and HTN in psoriasis patients with concurrent PsA. This is consistent with other studies suggesting an increased prevalence of metabolic syndrome in $\mathrm{PsA}^{20,21}$. Frequently associated comorbid diseases include cardiovascular (CV) disease, depression, anxiety, and inflammatory bowel disease ${ }^{7}$. Earlier reports on $\mathrm{CV}$ mortality in patients with PsA have shown an association by disease severity ${ }^{22}$, but the prevalence of $\mathrm{CV}$ risk factors (i.e., HTN, diabetes, and smoking) did not significantly differ across disease severities in our study.

The prevalence of PsA in patients with psoriasis has been inconsistently reported in previous studies. For example, Mease, et $^{\mathrm{a}} \mathrm{l}^{3}$ found a PsA prevalence of $30 \%$ in patients with psoriasis, and reported that up to $41 \%$ of these patients were previously undiagnosed. Larger population-based studies have found prevalence estimates as low as $8 \%{ }^{6}$. In our study, the prevalence of PsA among patients with psoriasis ranged from 16.3 to $47.9 \%$, depending on the severity of cutaneous psoriasis.
Several limitations apply to the interpretation of these findings. Existing data on the prevalence of psoriasis in patients with PsA are scarce, and while we found that 1 in 3 patients with PsA did not appear to have cutaneous psoriasis, the true prevalence of psoriasis among patients with PsA may be much higher. Indeed, we used pharmacotherapy as a measure of disease severity. However, treatment of PsA (e.g., with biologics or MTX) may also have beneficial effects on cutaneous psoriasis, thus reducing the measured disease severity in our study. While we limited the scope of our analyses to patients with PsA diagnosed by a rheumatologist, the diagnostic codes for PsA have to our knowledge not been validated, and misclassification could have occurred. Moreover, we lacked data on clinical and radiological measures, and it is likely that some patients may not seek medical treatment for their psoriasis or PsA. In addition, data regarding use of medical resources such as annual primary physician and specialists visits as well as hospitalizations were not included in descriptions of the disease burden. Lastly, the Danish population is predominantly of European descent, and extrapolation to other racial groups or ethnicities may be limited.

We found a higher psoriasis prevalence among patients with severe PsA and vice versa. The prevalence of comorbidity and the overall medical and psychiatric burden of disease is substantial in patients with psoriasis and PsA, and while 1 in 3 patients with PsA did not appear to have cutaneous symptoms in this population, presence of such symptoms may be underreported.

\section{REFERENCES}

1. Christophers E. Psoriasis - epidemiology and clinical spectrum. Clin Exp Dermatol 2001;26:314-20.

2. Ritchlin CT, Colbert RA, Gladman DD. Psoriatic arthritis. N Engl J Med 2017;376:2094-6.

3. Mease PJ, Gladman DD, Papp KA, Khraishi MM, Thaçi D, Behrens F, et al. Prevalence of rheumatologist-diagnosed psoriatic arthritis in patients with psoriasis in European/North American dermatology clinics. J Am Acad Dermatol 2013;69:729-35.

4. Moll JM, Wright V. Psoriatic arthritis. Semin Arthritis Rheum 1973;3:55-78.

5. Egeberg A, Kristensen LE, Thyssen JP, Gislason GH, Gottlieb AB, Coates LC, et al. Incidence and prevalence of psoriatic arthritis in Denmark: A nationwide register linkage study. Ann Rheum Dis 2017;76:1591-7.

6. Ogdie A, Langan S, Love T, Haynes K, Shin D, Seminara N, et al Prevalence and treatment patterns of psoriatic arthritis in the UK. Rheumatology 2013;52:568-75.

7. Ogdie A, Schwartzman S, Husni ME. Recognizing and managing comorbidities in psoriatic arthritis. Curr Opin Rheumatol 2015;27:118-26.

8. Ogdie A, Weiss P. The epidemiology of psoriatic arthritis. Rheum Dis Clin North Am 2015;41:545-68.

9. Schmidt M, Pedersen L, Sorensen HT. The Danish Civil Registration system as a tool in epidemiology. Eur J Epidemiol 2014;29:541-9.

10. Andersen TF, Madsen M, Jorgensen J, Mellemkjoer L, Olsen JH. The Danish National Hospital Register. A valuable source of data for modern health sciences. Dan Med Bull 1999;46:263-8.

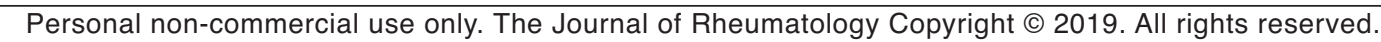


11. Kildemoes HW, Sorensen HT, Hallas J. The Danish National Prescription Registry. Scand J Public Health 2011;39:38-41.

12. Baadsgaard M, Quitzau J. Danish registers on personal income and transfer payments. Scand J Public Health 2011;39:103-5.

13. Danmarks Statistik. Health care. Visits to physicians. [Internet. Accessed January 10, 2019. Document in Danish.] Available from: www.dst.dk/da/Statistik/dokumentation/Times/sygesikringsstatistik

14. Egeberg A, Gyldenlove M, Zachariae C, Skov L. Validation of psoriasis severity classification based on use of topical or systemic treatment. J Eur Acad Dermatol Venereol 2018;32:e4-e5.

15. Egeberg A, Mallbris L, Gislason GH, Skov L, Hansen PR. Risk of multiple sclerosis in patients with psoriasis: A Danish nationwide cohort study. J Invest Dermatol 2016;136:93-8.

16. Ahlehoff O, Gislason GH, Jorgensen CH, Lindhardsen J, Charlot M, Olesen JB, et al. Psoriasis and risk of atrial fibrillation and ischaemic stroke: A Danish nationwide cohort study. Eur Heart J 2012;33:2054-64.

17. Zisman D, Eder L, Elias M, Laor A, Bitterman H, Rozenbaum M, et al. Clinical and demographic characteristics of patients with psoriatic arthritis in northern Israel. Rheumatol Int 2012; 32:595-600.
18. Husted JA, Thavaneswaran A, Chandran V, Gladman DD. Incremental effects of comorbidity on quality of life in patients with psoriatic arthritis. J Rheumatol 2013;40:1349-56.

19. Eder L, Shanmugarajah S, Thavaneswaran A, Chandran V, Rosen $\mathrm{CF}$, Cook RJ, et al. The association between smoking and the development of psoriatic arthritis among psoriasis patients. Ann Rheum Dis 2012;71:219-24.

20. Bostoen J, Van Praet L, Brochez L, Mielants H, Lambert J. A cross-sectional study on the prevalence of metabolic syndrome in psoriasis compared to psoriatic arthritis. J Eur Acad Dermatol Venereol 2014;28:507-11.

21. Caso F, Del Puente A, Oliviero F, Peluso R, Girolimetto N, Bottiglieri $\mathrm{P}$, et al. Metabolic syndrome in psoriatic arthritis: The interplay with cutaneous involvement. Evidences from literature and a recent cross-sectional study. Clin Rheumatol 2018;37:579-86.

22. Jamnitski A, Symmons D, Peters MJ, Sattar N, McInnes I, Nurmohamed MT. Cardiovascular comorbidities in patients with psoriatic arthritis: a systematic review. Ann Rheum Dis 2013;72:211-6. 